\title{
Ultrasound and Microbubbles for Coronary Artery Recanalization in Acute Myocardial Infarction
}

\author{
Bruno Garcia Tavares 1,2, Jeane Mike Tsutsui 1,2, Miguel Osman Aguiar1,2, Diego Ribeiro Garcia ${ }^{1,2}$, Múcio Tavares \\ Oliveira ${ }^{1}$, Alexandre Soeiro ${ }^{1}$, Pedro A Lemos-Neto ${ }^{1}$, Roberto Kalil-Filho ${ }^{1}$, Thomas Richard Porter ${ }^{3}$, Wilson Mathias-Jr ${ }^{1,2}$ \\ Instituto do Coração da Faculdade de Medicina da Universidade de São Paulo', São Paulo, SP; Grupo Fleury² - Brasil; Universidade de \\ Nebraska Medical Center ${ }^{3}$, Omaha - EUA
}

\section{Summary}

Background: Intravenous microbubbles (MB) and transthoracic ultrasound (US) have been utilized to recanalize epicardial vessels in animal models of ST segment elevation myocardial infarction (STEMI). The feasibility of such an ultrasound-guided approach in humans with STEMI have not been studied.

Objective: Pilot study with the aim to evaluate the efficacy of MB plus US on coronary artery recanalization rate in patients with STEMI.

Methods: Twenty-four patients (18 men, mean age $58 \pm 9$ years) admitted to the emergency room with STEMI were randomized into 3 groups. Patients either received MB plus custom designed high mechanical index (MI) impulses at 4-20 usec pulse duration ( $n=7)$, MB plus diagnostic high $M I(M I=1.0)$ with multiple impulses $<2$ usec pulse duration $(n=8)$ or $M B$ plus limited diagnostic high $\mathrm{MI}$ impulses $(<5)$ just to analyze myocardial perfusion, control group $(n=9)$. MB utilized in the study consisted of a solution of Definity $3 \%$. All randomized groups underwent emergent PCI.

Results: The mean door-to-balloon time were $76 \pm 35$ minutes in group US 4-20 usec, $70 \pm 20$ minutes in group US multiple impulses and $81 \pm 13$ minutes in control group $(p=N S)$. Angiographic recanalization before PCI was observed in $75 \%$ of patients treated with US multiple impulses, in $43 \%$ for US $4-20$ usec and $11 \%$ in control $(p=<0.05)$.

Conclusion: Utilization of MB and diagnostic US with multiple impulses may be a method of achieving early recanalization in acute STEMI. (Arq Bras Cardiol: Imagem cardiovasc. 2016;29(3):92-98)

Keywords: Myocardial Infarction; Ultrasonography; Microbubbles; Thrombolytic Therapy.

\section{Introduction}

Microbubbles are small gas-filled microspheres with acoustic properties that make them very useful as ultrasound contrast agents for diagnostic imaging. Diagnostic contrast echocardiography, supported by ultrasound and microbubbles, has been used to improve endocardial borders and analyze myocardial perfusion. ${ }^{1,2}$ The application of high mechanical index (MI) pulses through diagnostic transducers may result in cavitation of microbubbles and allow the analysis of backfilling of myocardial perfusion contrast, facilitating the assessment of myocardial perfusion. ${ }^{3-6}$ Additionally, the destruction of microbubbles by ultrasound may have therapeutic applications, such as drug delivery to specific locations or to accelerate the dissolution of thrombi, also known as sonothrombolysis.

Mailing Address: Jeane Mike Tsutsui •

Instituto do Coração (InCor)- HCFMUSP

Av. Dr. Enéas de Carvalho Aguiar, 44. Postal Code 05403-000, São Paulo, SP - Brazil

E-mail: jeane.tsutsui@incor.usp.br

Manuscript received April 24, 2016; revised May 05, 2016; accepted June 09, 2016.

DOI: $10.5935 / 2318-8219.20160024$
Current recanalization therapies in patients with acute myocardial infarction with ST-segment elevation myocardial infarction (STEMI) include pharmacological thrombolysis and percutaneous coronary intervention $(\mathrm{PCl})$, both with proven improvement of prognosis in patients with STEMI. ${ }^{7,8}$ But each of these therapies have significant limitations in clinical practice. Delay in time between the admission and arterial dilatation still exists in developed countries, during which myocardial necrosis can occur. This issue is even greater in developing countries, where access to primary $\mathrm{PCl}$ or even lytic therapy is jeopardized. In this context, the application of ultrasound and microbubbles in patients with STEMI can be a very promising treatment. Pre-clinical studies with pigs have shown that during a continuous intravenous infusion of perfluorocarbons-filled microbubbles, the ultrasonic energy issued by a diagnostic ultrasound transducer was able to restore the flow of microcirculation and improve the recanalization rates in experimental models of coronary arteries with acute thrombotic occlusion..$^{9,10}$

The purpose of this pilot study was to evaluate the effectiveness of microbubbles (MB) and ultrasound (US) on initial recanalization rates of the coronary artery in patients with STEMI. 


\section{Method}

\section{Study population}

Patients admitted to the emergency room with chest pain and evidence of STEMI to the 12-lead electrocardiogram (ECG) were invited to take part in the study. Inclusion criteria: Age $\geq 18$ years; eligible for emergency percutaneous coronary angioplasty; suitable apical and/or parasternal echocardiographic image; no known or suspected contraindication to the ultrasound contrast agent used in the study. Exclusion criteria: cardiogenic shock; life expectancy of less than two months or terminally ill patients history of previous myocardial infarction or severe heart disease, such as heart failure with ejection fraction $<40 \%$ in previous evaluation; severe valvar lesions with significant hemodynamic repercussions; known hemorrhagic diathesis or contraindication to inhibitors of glycoprotein $2 b / 3 a$, anticoagulants or aspirin; known right/left shunt or severe pulmonary hypertension; women of childbearing age. The project was approved by the Commission of Ethics in Institutional Research (CAPPesq 342.799), and all the patients or their relatives signed the informed consent form.

\section{Study protocol}

Patients were randomized into 3 groups (Figure 1):

- Microbubbles group + high mechanical index (MI) impulses at 4 - $20 \mu$ seg: patients received ultrasound customized for this protocol through a 1.7 MHz-S5/1 Philips transducer, $\mathrm{Ml}$ image $>1.0$ and $4-20 \mu$ seg pulse duration, applied inside the risk area during continuous infusion of microbubbles (Definity ${ }^{\circledR} 3 \%$, Lantheus Medical Imaging);

- Microbubbles group + high MI multiple impulses: patients received diagnostic ultrasound with high $\mathrm{MI}(>1.0)$ multiple impulses, 15 frames each, at short duration ( $2 \mu \mathrm{seg})$, applied inside and outside the risk area during continuous infusion of microbubbles (Definity ${ }^{\circledR} 3 \%$ );

Echocardiographies were performed with IE 33 Philips Medical Systems. Both groups used real-time perfusion imaging with low MI to evaluate microbubbles in the microvasculature. All patients were treated before and immediately after $\mathrm{PCl}$ for 50 to 60 minutes. The sonothrombolysis was performed while the patient waited to be taken to the hemodynamics room, without delay in the door-to-balloon time in view of the study protocol.

- Control group: the patients received microbubbles (Definity $3 \%$ ) and real-time perfusion imaging with low MI was taken only to evaluate perfusion within the risk area at certain intervals. All patients in control group also underwent $\mathrm{PCl}$.

A software specific for real-time myocardial perfusion imaging took echocardiographic images of longitudinal apical four-chamber and two-chamber views. The images were adjusted to minimize artifacts due to cardiac mobility and included: low MI (usually 0,2), pulse repetition frequency around $25 \mathrm{~Hz}$, and maximum line density selected to achieve a clear myocardial opacification. A sequence of ultrasonic pulses of $\mathrm{Ml}$ greater than 1.0 A (impulse) was manually triggered at the peak contrast intensity to destroy microbubbles within the myocardium. Then, low MI images were taken of at least 15 consecutive cardiac cycles to allow subsequent myocardial backfill.

\section{Echocardiographic and angiographic analysis}

The ejection fraction and the left ventricular end-diastolic and end-systolic volumes were calculated using Simpson's rule. The segmental wall motion score index was calculated by the sum of motility scores for every left ventricular segment $(1=$ normal, $2=$ hypokinesis, $3=$ akinesis and $4=$ dyskinesis) divided by the number of segments evaluated. The myocardial perfusion index was calculated by the sum of myocardial perfusion scores for every left ventricular segment ( 1 = normal, 2 = hypokinesis, $3=$ akinesis and $3=$ dyskinesis $)$ divided by the number of segments evaluated.

Coronary angiography was performed following standard protocol, and arterial patency was determined by analysis of the flow in the infarct-related vessel, before angioplasty. TIMI 0 or TIMI 1 cases were defined as occluded artery, and TIMI 2 or 3 were considered to represent an open artery.

Angiographic and echocardiographic analyzes were performed by interventional cardiologists and experienced echocardiographers who were not aware of the study's randomization.

\section{Statistical analysis}

Data are reported as mean \pm standard deviation for normally distributed variables and as a percentage for qualitative variables. The method ANOVA was applied to compare door-to-balloon time, duration of chest pain, and recanalization rates obtained immediately after angioplasty, between the three study groups. $\mathrm{P}$ values $<0.05$ were considered significant.

\section{Results}

For one year, a total of 24 patients (mean age $58 \pm 9$ years) admitted to the emergency room with STEMI were evaluated. Of these, $75 \%$ were male, $62 \%$ were smokers, $50 \%$ had a history of dyslipidemia, 50\% were hypertensive, and $38 \%$ were diabetic. Patients were randomized into the following groups: 7 patients in the 4-20 $\mu$ seg high MI group, 8 patients in the high $\mathrm{MI}$ group with multiple impulses, and 9 patients in the control group. There was no difference in duration of chest pain time of the three groups, and any delay in door-to-balloon time was observed in the three groups (Table 1).

The echocardiographic evaluation data of the patients under study are described in Table 2 .

The recanalization angiographic rate at the beginning of angiography (before performing $\mathrm{PCI}$ ) was 6 to 8 patients $(75 \%)$ in the group that received $\mathrm{MB}$ and US with high $\mathrm{MI}$ and multiple pulses; 3 in 7 patients (43\%) in the group that received $\mathrm{MB}$ and US with high $\mathrm{MI}$ and 4-20 $\mu$ seg pulse duration, and 1 in 9 patients (11\%) in the control group $(p<0.05)$, as shown in Figure 2. 


\section{Original Article}

Table 1 - Duration of chest pain and door-to-balloon time in the 3 groups

\begin{tabular}{lcc}
\hline & Door-to-balloon time (minutes) & Duration of chest pain (hours) \\
\hline High MI 4-20 $\mu$ seg $(n=7)$ & $76 \pm 35$ & $3: 28$ \\
High MI multiple impulses $(n=8)$ & $70 \pm 20$ & $3: 30$ \\
Control $(n=9)$ & $81 \pm 13$ & $3: 25$ \\
$p$ & $n s$ & $n s$ \\
\hline
\end{tabular}

Table 2 - Echocardiographic characteristics of left ventricular function

\begin{tabular}{|c|c|c|c|c|c|c|}
\hline Group & LVEF (\%) & LV-EDV (mL) & LV-ESV (mL) & WMSI & MPI & $\begin{array}{l}\text { Number of segments with } \\
\text { perfusion defect }\end{array}$ \\
\hline Control & 49 & 93 & 47.5 & 2 & 2 & 9 \\
\hline Control & 48 & 125 & 65 & 2.23 & 2.29 & 15 \\
\hline Control & 34 & 180 & 118 & 2.7 & 2.56 & 16 \\
\hline Control & 42 & 71 & 41 & 2.05 & 2 & 11 \\
\hline Control & 25 & 142 & 107 & 2.35 & 2.29 & 14 \\
\hline Control & 49 & 65 & 33 & 1.64 & 1.41 & 7 \\
\hline Control & 32 & 144 & 108 & 2.47 & 2.29 & 15 \\
\hline Control & 39 & 80 & 49 & 2.11 & 2 & 12 \\
\hline Control & 41 & 83 & 49 & 1.94 & 1.93 & 9 \\
\hline High Ml 4-20 $\mu$ seg & 36 & 135 & 87 & 2.17 & 2.05 & 11 \\
\hline High Ml 4-20 $\mu$ seg & 47 & 74 & 39.5 & 2.05 & 1.88 & 9 \\
\hline High Ml 4-20 $\mu$ seg & 55 & 84 & 38 & 1.76 & 1.12 & 2 \\
\hline High Ml 4-20 $\mu$ seg & 57 & 80 & 34.5 & 1.7 & 1.41 & 6 \\
\hline High Ml 4-20 $\mu$ seg & 54 & 87 & 40 & 1.41 & 1.41 & 7 \\
\hline High Ml 4-20 $\mu$ seg & 46 & 48 & 26 & 2.05 & 1.82 & 9 \\
\hline High Ml 4-20 $\mu$ seg & 64 & 106 & 38 & 1.17 & 1.23 & 4 \\
\hline High MI multiple impulses & 41 & 113 & 67 & 1.76 & 2.17 & 12 \\
\hline High MI multiple impulses & 41 & 92 & 54 & 2.05 & 1.86 & 7 \\
\hline High MI multiple impulses & 35 & 156 & 141 & 2.35 & 2.23 & 14 \\
\hline High MI multiple impulses & 35 & 103 & 67 & 2.05 & 2.16 & 12 \\
\hline High MI multiple impulses & 43 & 120 & 68 & 2.41 & 2.37 & 13 \\
\hline High MI multiple impulses & 57 & 90 & 39 & 1.23 & 1.47 & 5 \\
\hline High MI multiple impulses & 63 & 46 & 17 & 1.23 & 1.17 & 3 \\
\hline High MI multiple impulses & 62 & 60 & 23 & 1.17 & 1.17 & 3 \\
\hline
\end{tabular}

LVEF: left ventricular ejection fraction; LV-EDV: left ventricular end-diastolic volume; LV-ESV: left ventricular end-systolic volume; WMSI: segmental wall motion score index; MPI: myocardial perfusion index.

Example description: 57-year male patient with a history of smoking (40 cigarettes/day) and dyslipidemia; he was admitted to the emergency room with chest pain frame intensity $9(0-10)$, radiating to the left arm, lasting $1 \mathrm{~h} 30$. His ECG at admission showed ST-segment elevation on the side wall (Figure 3A). The patient signed the informed consent form, underwent echocardiography with perfusion that showed akinesia of the inferior and septal wall associated with myocardial perfusion defect (Figure 4A). The patient was treated with Definity ${ }^{\circledR}$ through peripheral intravenous access and US with high MI with multiple impulses for 15 minutes while waiting for the $\mathrm{PCl}$. In the hemodynamics room, prior to coronary angiography, the patient showed improvement of chest pain and a slight reduction of ST-segment elevation on ECG (Figure 3B). Angiography showed right coronary artery with subocclusive lesion with thrombus, rechanneled (TIMI 3 flow, Figure 5A). The patient underwent implantation of intracoronary stent 


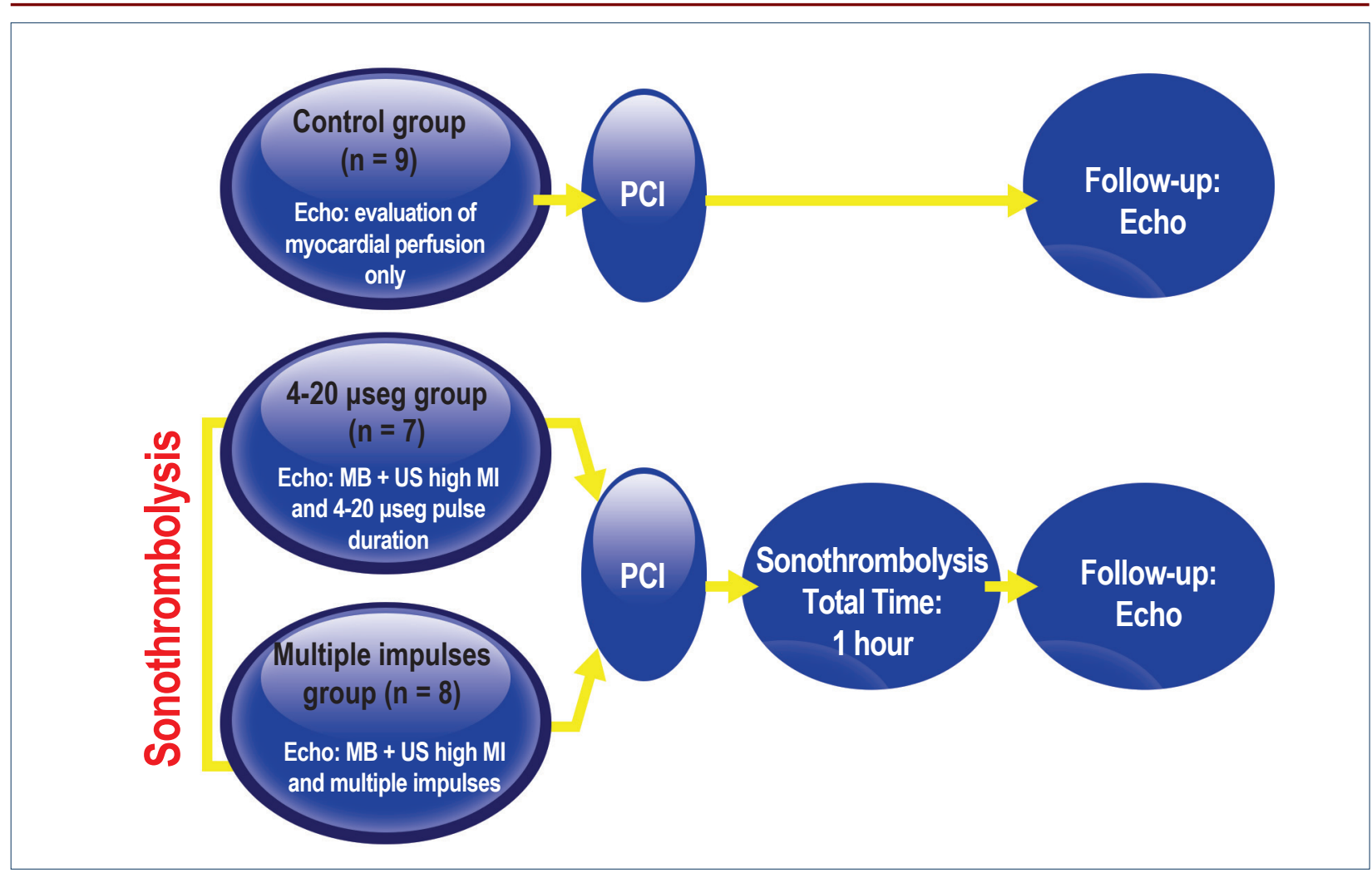

Figure 1 - Flowchart of randomization of the 3 groups. MB: microbubbles; US: ultrasound; MI: mechanical index; PCl: percutaneous coronary intervention.

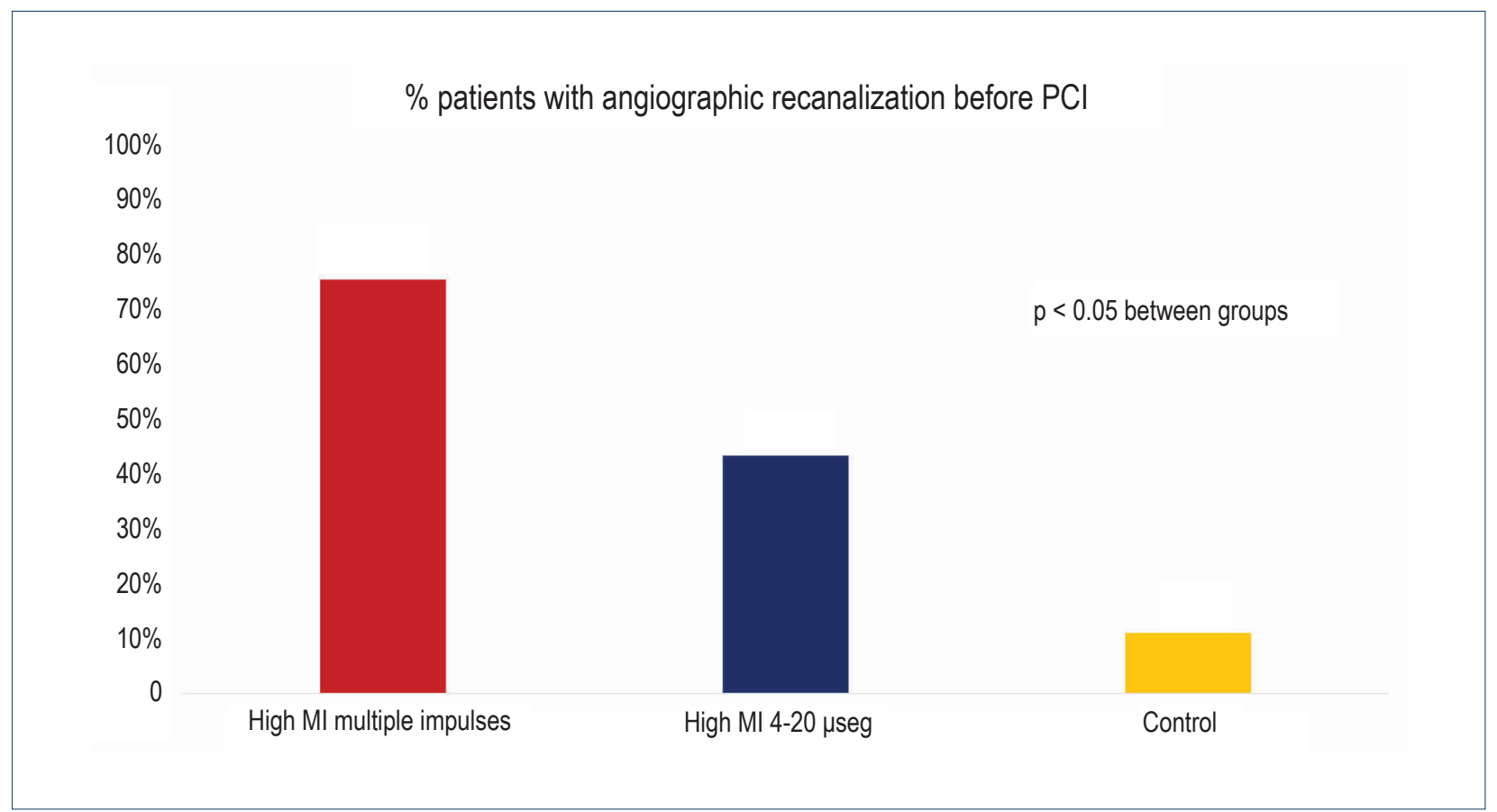

Figure 2 - Angiographic recanalization rate observed before the first angiography, before percutaneous coronary intervention (PCl) in the 3 study groups. 


\section{Original Article}

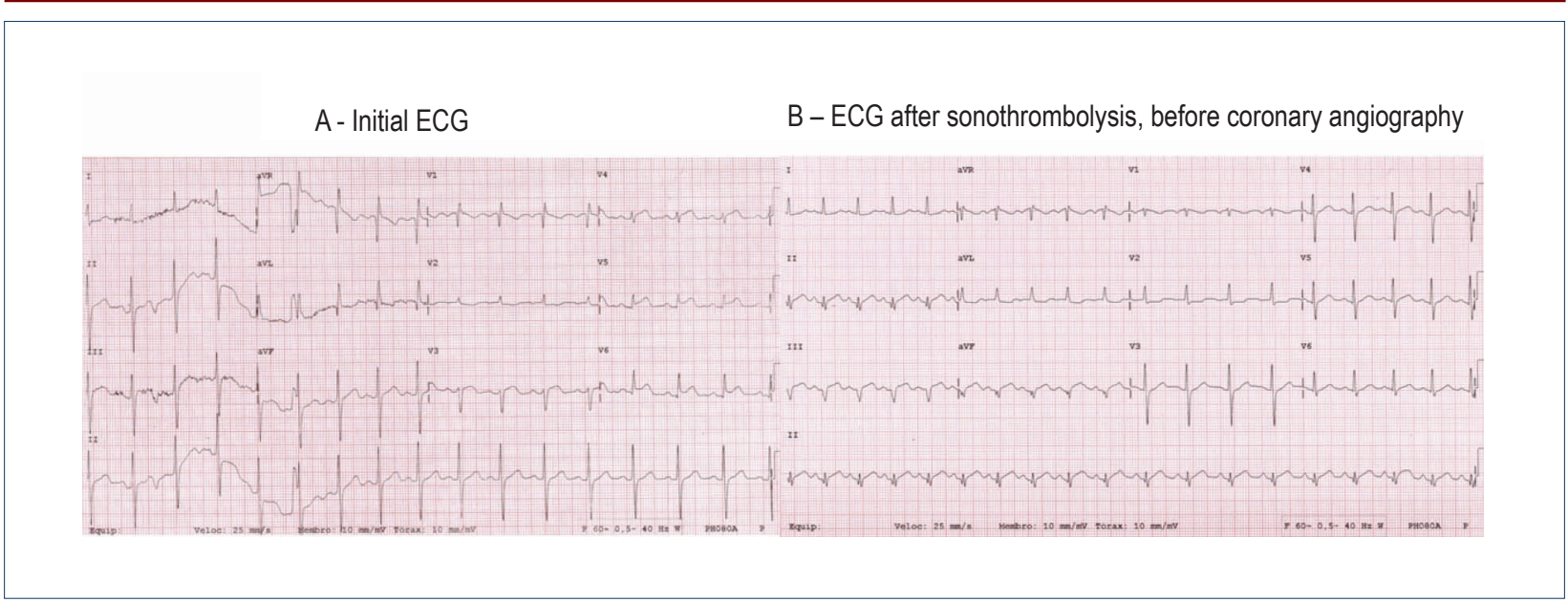

Figure 3 - 12-lead electrocardiogram (ECG) traces at admission to the emergency room (A) showing ST-segment elevation on the side wall, and after 15 minutes of sonothrombolysis, before coronary angiography, with a slight reduction in ST-segment elevation (B).

A - ECG with myocardial perfusion before sonothrombolysis

\section{B - ECG with myocardial perfusion after sono- thrombolysis}

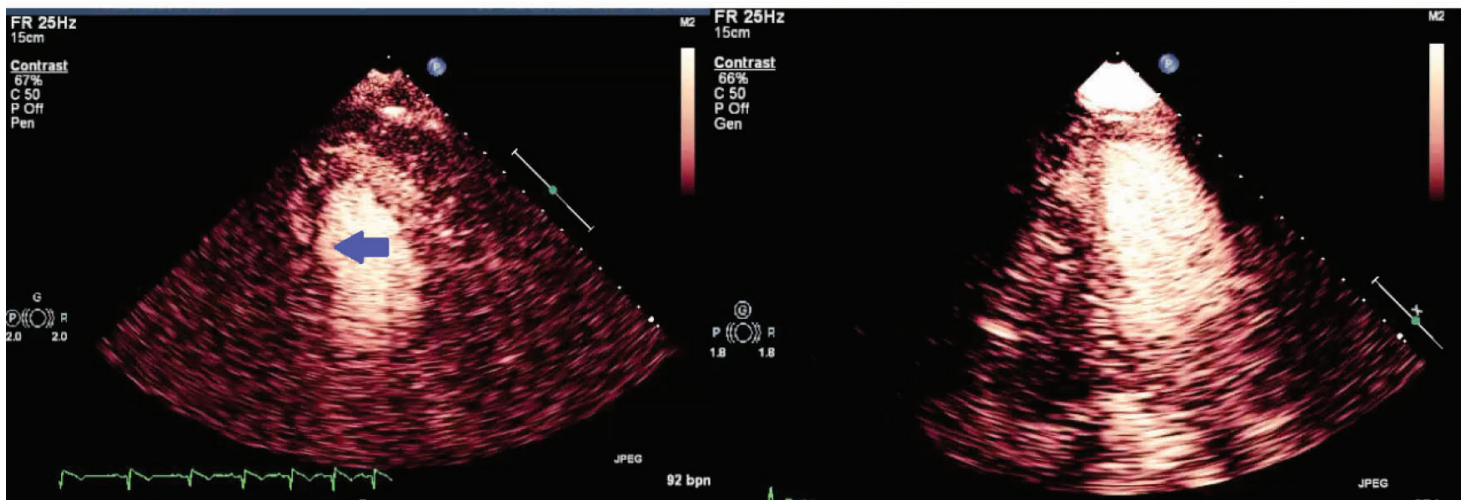

Figure 4 - Real-time perfusion ECG imaging of apical two-chambers view, showing akinesia of the inferior wall associated with perfusion defect (A, arrow). After 15 minutes of sonothrombolysis, prior to coronary angiography, the echocardiographic evaluation showed that the inferior akinesia remained, but the myocardial perfusion defect on this wall improved $(B)$.

(Figure 5B). After the angioplasty, the patient remained under sonothrombolysis therapy until the end of the treatment. The post-treatment ECG shows improvement in myocardial perfusion in the infarcted area, and maintenance of the change in segmental contractility (Figure 4B), with a slight decrease in left ventricular global function (ejection fraction 55\%)

\section{Discussion}

Today, epicardial recanalization is the main therapy for STEMI. Although $\mathrm{PCl}$ remains the first-line treatment for STEMI patients, in Brazil, only a small number of people have access to this therapy. Nevertheless, in patients in whom epicardial recanalization is successful through emergency $\mathrm{PCl}$, the distal microvasculature to the occluded vessel remains closed, leading to significant myocardial necrosis in up to $65 \%$ of patients. ${ }^{11,12}$

The high-energy transthoracic ultrasound has been studied both as a supporting therapy for fibrinolytic drugs in the treatment of arterial thrombi, and a single method for the treatment of vascular thrombi. ${ }^{13-17} \mathrm{~A}$ proposed mechanism of how the ultrasound dissolves the thrombus is by inducing cavitation. ${ }^{18,19}$ Cavitation is the ultrasonic generation of gas bodies that expand and retract. This leads to shear forces, which disturb the environment and have the potential to break thrombus. 


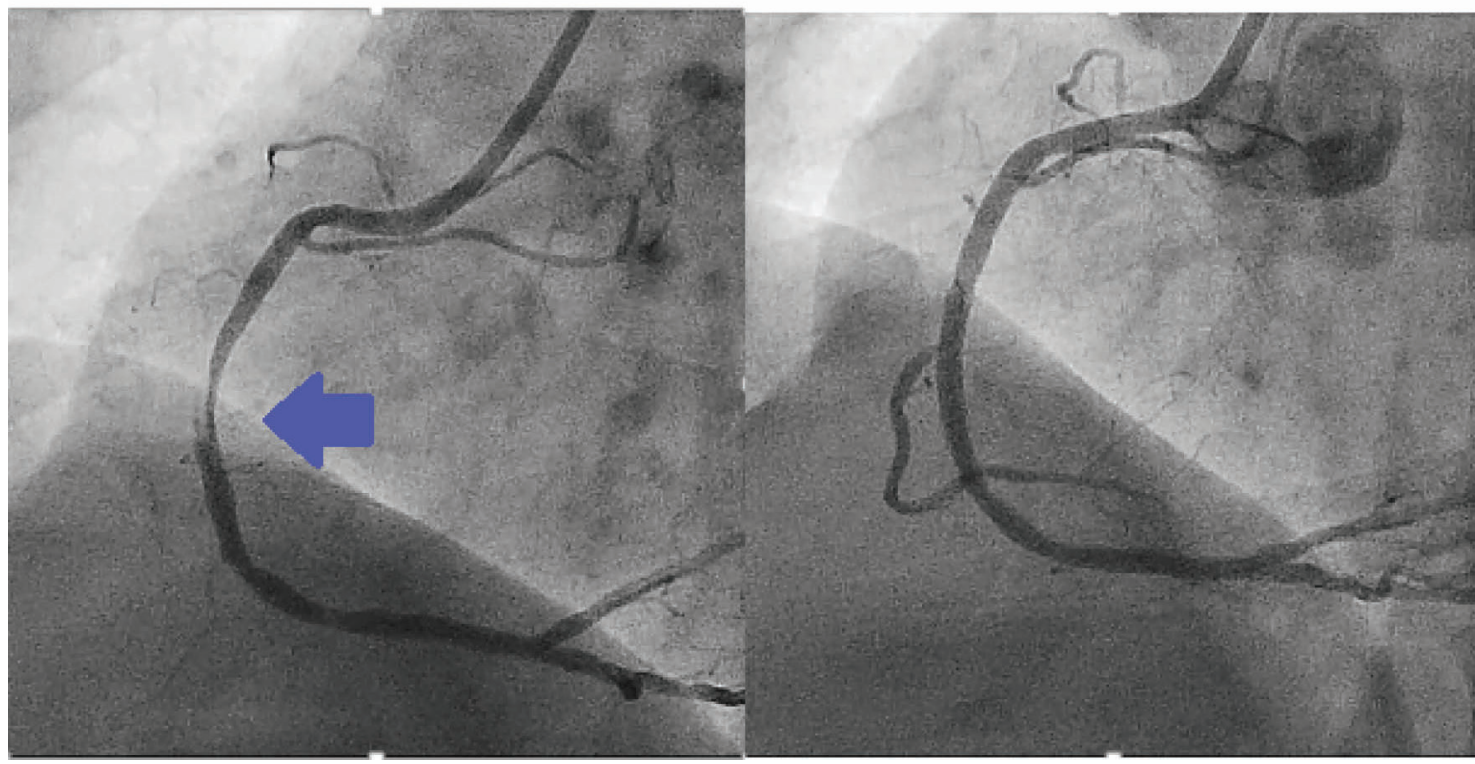

Figure 5 - Coronary angiographic images after 15 minutes of sonothrombolysis showing subocclusion with thrombus in the middle third of the right coronary artery (A, arrow), with the presence TIMI 3 filling flow. The patient underwent implantation of intracoronary stent (B).

\section{Future application}

The demonstration that the use of microbubbles, originally applied for diagnostic ultrasound, also has effect on the restoration of the affected epicardial coronary flow and microcirculation in STEMI will drastically impact how patients are handled in the initial phase of treatment. In view of the widespread availability and safety of ultrasound and microbubbles, the therapy to restore blood flow could be started in primary care units and in ambulances by skilled technicians as soon as the diagnosis of STEMI was defined. This would lead to a significant decrease in cardiac muscle losses, which occur while waiting for the safe performance of emergency primary angioplasty, besides reducing future complications to the patient, such as development of heart failure and complex arrhythmias.

\section{Limitations}

This is a pilot study, therefore, results in a greater number of patients, and the analysis of other myocardial perfusion parameters to define the impact on the microvasculature are still required.

\section{Conclusion}

This pilot study proved that it is possible to treat STEMI patients with microbubbles and ultrasound in the emergency room. The acoustic radiation forces generated by multiple impulses of diagnostic US of high MI applied on the myocardium can be a method of early recanalization of epicardial arteries in STEMI patients.

\section{Authors' contributions}

Research conception and design: Tsutsui JM, Kalil-Filho R, Porter TR, Mathias-Jr W; Data collection: Tavares BG, Aguiar MO, Garcia DR, Soeiro A; Data analysis and interpretation: Tavares BG, Aguiar MO, Garcia DR, Lemos-Neto PA; Statistical analysis: Tsutsui JM; Obtaining funding: Porter TR, Mathias-Jr W; Manuscript writing: Tavares BG, Soeiro A, Lemos-Neto PA; Critical revision of the manuscript's major intellectual content: Tsutsui JM, Kalil-Filho R, Porter TR, Mathias-Jr W; Clinical follow-up of patients with acute myocardial infarction: Oliveira MT.

\section{Potential Conflicts of Interest}

No relevant conflicts of interest.

\section{Sources of Funding}

This study was funded by Fundação de Amparo à Pesquisa do Estado de São Paulo (Fapesp) (project 2010/52114-1)

\section{Academic Association}

This manuscript is part of the dissertation (Master's degree, Doctoral degree, habilitation) of Bruno Garcia Tavares from the Instituto do Coração - Hospital das Clínicas of Faculdade de Medicina, Universidade de São Paulo. 


\section{References}

1. Mulvagh S, Rakowski H, Vannan MA, Abdelmoneim SS, Becher H, Bierig M, et al. American Society of Echocardiography Consensus Statement on the Clinical Applications of Ultrasonic Contrast Agents in Echocardiography. J Am Soc Echocardiogr. 2008;21(11):1179-201.

2. Porter TR, Abdelmoneim S, Belcik JT, McCulloch ML, Mulvagh SL, Olson $J$, et al. Guidelines for the cardiac sonographer in the performance of contrast echocardiography: a focused update from the American Society of Echocardiography. J Am Soc Echocardiogr. 2014;27(8):797-810.

3. Shimoni S, Zhoghbi WA, Xie F, Kricsfeld D, Iskander S, Gobar L, et al. Realtime assessment of myocardial perfusion and wall motion during bycicle and treadmill exercise echocardiography: comparison with single photon emission computed tomography. J Am Coll Cardiol. 2001;37(3):741-7.

4. Porter TR, Xie F. Myocardial perfusion imaging with contrast ultrasound. JACC Cardiovasc Imaging. 2010;3(2):176-87.

5. Wei K, Jayaweera AR, Firoozan S, Linka A, Skyba DM, Kaul S. Quantification of myocardial blood flow with ultrasound-induced destruction of microbubbles administered as a constant venous infusion. Circulation. 1998;97(5):473-83.

6. Vogel R, Indermuhle A, Reinhardt J, Meier P, Siegrist PT, Namdar M, et al. The quantification of absolute myocardial perfusion in humans by contrast echocardiography: algorithm and validation. J Am Coll Cardiol. 2005;45(5):754-62.

7. Pollack CV Jr, Braunwald E. 2007 Update to the ACC/AHA. Guidelines for the management of patients with unstable angina and non ST- segment elevation myocardial infarction: implications for Emergency Department Practice. Ann Emerg Med. 2008;51(5):591-606.

8. O'Gara PT, Kushner FG, Ascheim DD, Casey DEJr, Chung MK, de Lemos JA, et al. 2013 ACCF/AHA guideline for the management of ST-elevation myocardial infarction: a report of the American College of Cardiology Foundation/ American Heart Association Task Force on Practice Guidelines. Circulation. $2013 ; 127(4): 529-55$

9. Xie F, Lof JMS, Matsunaga T, Zutshi R, Porter TR. Diagnostic ultrasound combined with glycoprotein $2 \mathrm{~b} / 3 \mathrm{a}$ targeted microbubbles improve microvascular recovery following acute coronary thrombotic occlusions. Circulation. 2009;119(10):1378-85.
10. Xie F, Slikkerveer J, Gao S, Lof J, Kamp O, Unger E, et . Coronary and microvasalcular thrombolysis with guided diagnostic ultrasound and microbubbles in acute ST segment elevation myocardial infarction. J Am Soc Echocardiogr. 2011;24(12):1400-8.

11. Nicoli G, Burzotta F, Galiuto L, Crea F. Myocardial no-reflow in humans. J Am Coll Cardiol. 2009;54(4):281-92.

12. McLaughlin MG, Stone GW, Aymong E, Gardner G, Mehran R, Lansky AJ, et al. Prognostic utility of comparative methods for the assessment of ST-segment resolution after primary angioplasty for acute myocardial infarction: the Controlled Abciximab and Device Investigation to Lower Late Angioplasty Complications (CADILLAC) trial. J Am Coll Cardiol. 2004;44(6):1215-23.

13. Tachibana K, Tachibana S. Albumin microbubble echo-contrast material as an enhancer for ultrasound accelerated thrombolysis. Circulation. 1995;92(5):1148-50.

14. Porter TR, Leveen RF, Fox R, Kricsfeld A, Xie F. Thrombolytic enhancement with perfluorocoarbon-exposed sonicated dextrose albumin microbubbles. Am. Heart J. 1996;132(5):964-8.

15. Suchkova VN, Baggs RB, Francis CW. Effect of $40 \mathrm{KHz}$ ultrasound on acute thrombotic ischemia in a rabbit femoral artery thrombosis model: enhancement of thrombolysis and improvement in capillary muscle perfusion. Circulation. 2000;101(19):2296-301.

16. Dhond MR, Nguyen TT, Dolan C, Pulido G, Bommer WJ. Ultrasoundenhanced thrombolysis at $20 \mathrm{KHz}$ with air-filled and perfluorocarbon-filled contrast biospheres. J Am Soc Echocardiogr. 2000;13(11):1025-9.

17. Xie F, Tsutsui JM, Lof J, Unger EC, Johanning J, Culp WC, et al. Effectiveness of lipid microbubbles and ultrasound in declotting thrombosis. Ultrasound Med Biol. 2005;31(7):979-85.

18. Everbach EC, Francis CW. Cavitational mechanisms in ultrasoundaccelerated thrombolysis at $1 \mathrm{MHz}$. Ultrasound Med Biol. 2000;26(7):1153-60.

19. Prokop AF, Soltani A, Roy RA. Cavitational mechanisms in ultrasoundaccelerated fibrinolysis. Ultrasound Med Biol. 2007;33(6):924-33. 